\title{
Environmental enrichment improved cognitive deficits more in peri-adolescent than in adult rats after postnatal monosodium glutamate treatment
}

\author{
S Madhavadas', S Subramanian', BM Kutty ${ }^{2}$ \\ ${ }^{1}$ Department of Neurochemistry, National Institute of Mental Health and Neurosciences, Bangalore, India \\ ${ }^{2}$ Department of Neurophysiology, National Institute of Mental Health and Neurosciences, Bangalore, India
}

Received: November 2, 2016

Accepted: November 15, 2017

\begin{abstract}
Exposure to enriched environment (EE) is known to promote sensory, cognitive, and motor stimulation with intensified levels of novelty and complexity. In this study, we investigated the positive regulatory effect of short-term exposure to EE on establishing functional recovery in monosodium glutamate (MSG)-induced obese rats. Unless treated, MSG rats exhibited peripheral insulin resistance, cognitive deficits, and a reduction in the total hippocampal volume with decreased neuron count in the DG, CA3, and CA1 subfields. These MSG rats were exposed to shortterm $\mathrm{EE}$ for 15 days for a period of $6 \mathrm{~h}$ /day, beginning either at 45 or at 75 days of age. EE exposure has improved insulin sensitivity, yielded a significant increase in total hippocampal volume along with increase in neuron number in the CA1 subfield of the hippocampus in both age groups. However, as assessed by radial arm maze task, which relies upon the positive reinforcement to test spatial memory, and the Barnes maze task, which utilizes an aversive learning strategy, a complete recovery of cognitive function could be achieved in 2-month-old rats only and not among 3-month-old rats, thus highlighting the importance of critical window period for EE interventions in restoring the memory functions. These results suggest the therapeutic potential of EE paradigm in prevention of cognitive disorders.
\end{abstract}

Keywords: radial arm maze, barnes maze, hippocampus, spatial memory

\section{Introduction}

The prevalence of obesity has been alarmingly increasing worldwide, and, when left untreated, promoting a rise in serious consequences, particularly metabolic disorders and a rise in mortality. Convergent clinical studies have revealed a predictive longitudinal link between obesity and the development of age-related cognitive deficits and neurological diseases, such as Alzheimer's disease (AD) (13). In agreement with clinical studies, experimentally induced obesity is associated with a wide array of cognitive abnormalities including impairment in learning and memory (29). For example, performance of spatial learning and long-term memory $(4,7)$ or contextual fear conditioning $(27)$ is impaired in dietinduced obesity models (7).

Rodents treated neonatally with monosodium glutamate (MSG) develop into obese, stunted adults (45) and have been frequently studied as models for obesity (14). Reduced energy expenditure and sedentary behavior were assumed to be fueling the abnormal increase

\footnotetext{
Corresponding author: Sarada Subramanian

Department of Neurochemistry, National Institute of Mental Health and Neurosciences

Bangalore 560 029, India

Phone: +91 802699 5165; Fax: +91 802656 4830; E-mail: sarada@nimhans.ac.in
} 
of fat deposits in postnatal MSG-treated rats as they present with adult age obesity without hyperphagia (37). MSG induces obesity by disrupting the hypothalamic-signaling cascade of leptin action (37). The consequent leptin resistance in the central nervous system (CNS) results in the characteristic changes associated with $\mathrm{AD}(8)$. It has been shown earlier that MSG-treated obese rats exhibited significant decline in cognitive functions in peri-adolescent and adult stages $(23,32,35)$. Transition from the pre-obese to the obese state was found to occur in MSG-treated rats between the 30th and the 90th day (40). Therefore, to observe the obesity-associated metabolic disturbances further leading to cognitive impairment, this study was designed to investigate the early changes in the transition state of the pre-obese to obese state in the postnatal MSG-treated rats.

Neonatal MSG administration produces behavioral deficits, which were proved by many studies and a few have examined the possible ways of reversal following exposure to enriched environment (EE) $(24,52)$. A diverse range of molecular, cellular, and behavioral effects of EE has been described in wild-type mice and rats, as studied previously $(6,47)$. The EE-induced changes are not merely transcriptional and extend to effects on the proteome (25). The cellular effects of EE, which are presumably dependent on molecular changes, include enhanced adult neurogenesis (56) and synaptic plasticity (11). Furthermore, specific neuronal cell populations in the areas concerned with spatial information and cognitive map development, viz., the dentate gyrus (DG), CA1, and CA3 areas of hippocampus, subiculum, and entorhinal cortex (10) have been shown to be activated by EE (2) and these effects include glia (33) also. The time where there is a requirement for environmental input for proper development of a particular brain circuit is called a critical period (22). If the brain circuit is not stimulated, the function served by the brain circuit will be permanently compromised. These windows of plasticity are very important in brain development. However, a range of other cellular effects have been described, including those impacting on metabolism, the immune system (38) and the hypothalamic-pituitary-adrenal-axis (39). The EE-induced increase in adult hippocampal neurogenesis may contribute to enhancement of specific cognitive functions, in particular, pattern separation (53). As shown in previous studies, EE provides a useful and non-invasive method of reversing behavioral deficits in several disorders. Therefore, this study was aimed to investigate the efficacy of EE on behavioral recovery following neonatal MSG administration in 45- and 75-day old rats.

\section{Materials and Methods}

Monosodium L-glutamate (MSG), 5, 5-dithiobis-2-nitrobenzoic acid, butyrylthiocholine iodide (BTCI), glucose oxidase, peroxidase, cholesterol standard, 4-aminophenazone and thiobarbituric acid were purchased from Sigma-Aldrich, Bangalore. For the quantitative analysis of rat serum insulin, the ELISA kit was purchased from EMD Millipore, USA. All other reagents used were of analytical grade and obtained locally.

\section{Animals and experimental protocols}

A total of 128 male Sprague Dawley rat pups were chosen for the study. They were maintained under controlled temperature and light. Neonatal rat pups were injected with MSG (4 mg/g body weight) daily once for 14 consecutive days after birth through intraperitoneal route. Rats of the same age and strain, receiving saline, served as control. For the evaluation of the beneficial effects of exposure to $E E$ at 45 and 75 days of age, the rats were first exposed to socially enriched housing environment/EE before subjecting to the 
behavioral training in the radial arm maze (RAM) and Barnes maze and the rats were divided into the following groups ( $n=8$ in each group). Different sets of rats were used for different maze studies.

Group I - untreated control rats (Ctrl)

Group II - controls exposed to EE for 15 days (Ctrl EE)

Group III - MSG-treated rats (MSG)

Group IV - MSG rats exposed to EE for 15 days (MSG EE)

After the treatment, the rats attained the age of 2 and 3 months, respectively. Behavioral testing, biochemical analysis, and stereology experiments were performed with these rats. The study design is presented in Fig. 1. All the experimental rats survived till sacrifice. All the procedures were in compliance with the Institutional Animal Ethics Committee (CPCSEA registration no. of the institute: reg. no. 12/GO/ac/99/CPCSEA; IAEC approval no. AEC/43/ 258/NC dated April 21, 2011).

\section{Housing conditions}

Standard housing condition. About 2-3 rats were housed in each polypropylene cage $(36 \times 23 \times 15 \mathrm{~cm})$ containing paddy husk as bedding material, which was changed on alternate days. Food and water were provided ad libitum to all the groups of rats throughout the study. They were kept in a well-ventilated room and 12-h light-dark cycle was maintained.

EE housing condition. The EE paradigm we used in this study was self-defined by our laboratory, considering that the goal of EE is to provide rats with opportunities to express their full range of species-typical behavioral patterns, and a certain degree of control over their environment. Both the Ctrl EE and MSG EE groups were exposed to enriched housing conditions for $6 \mathrm{~h}$ daily (from 10:00 AM to 4:00 PM) for 15 days. Enriched housing was provided by exposing the rats to a specially designed cage with a dimension of $81.5 \times 61 \times$ $45 \mathrm{~cm}$, the walls made of metal wire mesh and the bottom with wooden platform. A sliding door was provided for replacing the paddy husk and placing the rats. About 8-10 rats were housed in this cage to provide social stimulation. The cage was equipped with various exploratory materials like plastic tunnels $(30-\mathrm{cm}$ long and $12 \mathrm{~cm}$ in diameter), metal

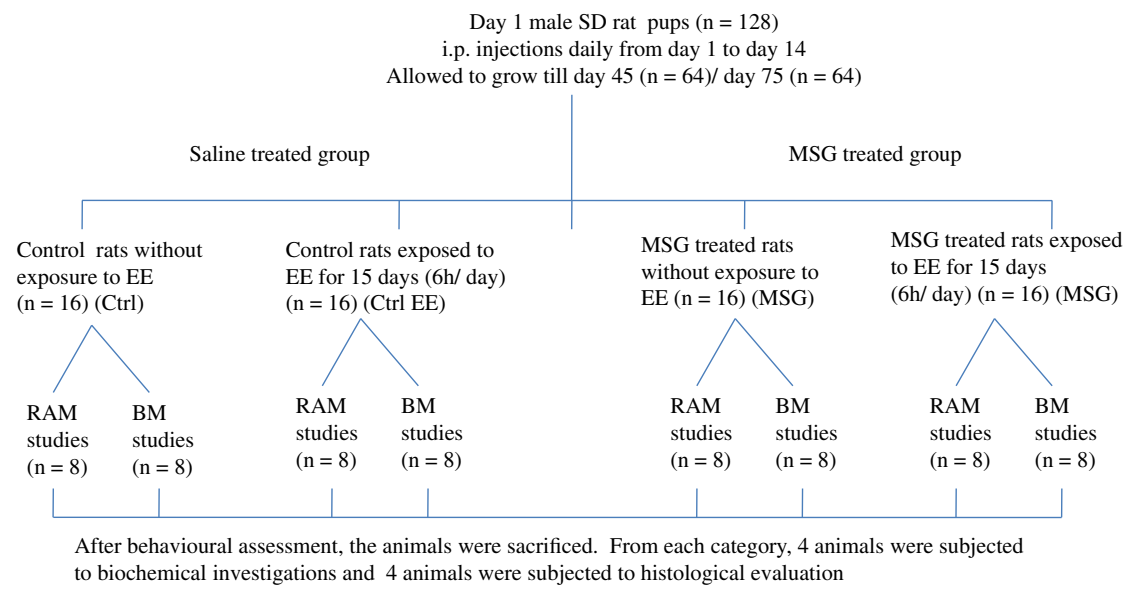

Fig. 1. The study design and the distribution of rats to various groups is indicated. RAM: radial arm maze; BM: Barnes maze 
platforms (40-cm long $\times 25$-cm width and $25-\mathrm{cm}$ long $\times 15-\mathrm{cm}$ width), balls, rattle, ladders, and toys of various sizes and textures (wood, metal, and plastics). The rats were exposed to novelty stimulation by changing the exploratory objects daily.

\section{RAM studies}

Four arm baited task. In this task, only four arms (arms 1, 3, 5, and 7) in the 8-arm maze were baited. At the start of each trial, the food reward was placed in the specified arms $(1,3,5$, and 7), which was kept constant across trials and sessions. To begin with each training trial, the rat was placed in the central compartment of the maze. All the arms were opened. The training trial continued until the rat retrieved the food reward from all the four arms or spent $5 \mathrm{~min}$ in the maze. Reentry into an arm was considered as working memory error. Entry into an unbaited arm was considered as reference memory error. The rats were given four trials of one session per day with an intertrial interval of $15 \mathrm{~min}$. The training continued until the rat learnt to retrieve the food reward from all the four arms.

Retention test. Retention test was carried out 10 days following acquisition. In the retention test, performance in a single session (four trials) was assessed; depending on 5 min allocated time to complete the task and the mean average performance of the four trials was calculated.

\section{Barnes maze}

The maze consisted of a gray circular platform with $120 \mathrm{~cm}$ diameter that was brightly lit from above. The platform was elevated $90 \mathrm{~cm}$ above the floor by a stand. Twelve holes measuring $6.25 \mathrm{~cm}$ in diameter were evenly spaced around the perimeter. A rectangular black escape box $(35-\mathrm{cm}$ length $\times 15-\mathrm{cm}$ wide $\times 19-\mathrm{cm}$ depth) was placed beneath the target hole. Four visual cues consisting of various shapes (triangle, square, circle, and vertical bar) were placed at evenly spaced intervals on the inside of the maze wall. Two 150-W lights were hung above the platform to create a potentially adverse environment and trigger the rats to escape from the brightly lit, open surface (in favor of the dark environment of the escape box). The escape box location remained constant for any individual rat across test trials during acquisition and retention. The Barnes maze task was carried out in accordance with our earlier study (36).

Retention test. After the acquisition trials, testing was abated for 5 days and retention was evaluated in a single session (three trials) depending on 2 min allocated time to reach the escape box and the mean average performance of the retention session was calculated.

After completing the behavioral assessment trials, four rats from each group were sacrificed by cervical dislocation for biochemical analysis and remaining rats were subjected to histological verification of hippocampal neurodegeneration. Approximately $1 \mathrm{ml}$ of blood was collected from each rat through cardiac puncture; serum was separated and stored at $-20{ }^{\circ} \mathrm{C}$ until analysis.

\section{Anthropometric measurements and analysis of the serum parameters}

The body weight and body length were used to confirm the body mass index (BMI) status in the rats as described earlier (35). The levels of glucose, cholesterol, and thiobarbituric acid reactive substances (TBARs) were estimated by the procedure detailed earlier (36). Briefly, glucose was estimated by glucose oxidase/peroxidase method, wherein, glucose present in the serum was oxidized by the enzyme glucose oxidase to gluconic acid. The liberated 
hydrogen peroxide was converted to water and oxygen by the enzyme peroxidise. Utilization of the released oxygen by 4-aminophenazone with phenol resulted in formation of pinkcolored product, which was measured at $510 \mathrm{~nm}$. Total cholesterol was estimated by first deproteinating the serum with ferric chloride-acetic acid reagent treatment. The protein free supernatant was treated with concentrated sulfuric acid. The reddish purple color developed was measured at $540 \mathrm{~nm}$. TBARs were extracted with glacial acetic acid and measured by spectrophotometry at $532 \mathrm{~nm}$. Levels of insulin in the serum were measured by sandwich ELISA method as per manufacturer's instructions. The sensitivity of the assay for insulin was $0.2 \mathrm{ng} / \mathrm{ml}$ and the detection range was $0.2-10 \mathrm{ng} / \mathrm{ml}$. The serum butyryl cholinesterase activity was measured by Ellman's method (17) using the artificial substrate BTCI and the enzyme activity was expressed as $\mathrm{U} / \mathrm{ml}$ serum.

\section{Histological assessment of hippocampal neurodegeneration}

Following behavioral studies, all rats from different groups were subjected to histological verification of hippocampal neurodegeneration. The volume analysis of total hippocampus as well as neuron count in the CA1, CA3, and DG subfields of the hippocampus was carried out. The rats were transcardially perfused, with $0.9 \%$ saline followed by $10 \%$ formalin. The brains were subsequently removed and post fixed in $10 \%$ formalin. About $30-\mu$ m-thick coronal sections were taken (every fifth section) (Vibratome VT1000S, Leica Germany) at the level of dorsal hippocampus [from 2.3 to $3.8 \mathrm{~mm}$ with reference to Bregma, using the Paxinos and Watson (48) rat brain atlas], stained with $0.1 \%$ cresyl violet and examined microscopically. Photomicrographs of the sections were taken using the Olympus BX61 microscope.

\section{Hippocampal volume analysis}

Hippocampal volume estimation using the Cavalieri principle was performed with StereoInvestigator software 8.1 (MBF Bioscience, Microbrightfield Inc., Williston, VT, USA) and Olympus BX61 microscope fitted with a motorized stage. Volume analysis was carried out as described in our previous study (34).

\section{Estimation of neuron number}

Series of sections, including every fifth section, were randomly chosen and coded to ensure blinding to the investigators. The sections used for volume estimation were used for the neuron number estimation also. Unbiased stereological estimates of neuron number were obtained with the commercial software Stereo-Investigator software 8.1 and Olympus BX61 microscope fitted with a motorized stage. Neuron number in both the hemispheres of all the experimental rats were estimated with the optical fractionators probe (20), using a $400 \times$ magnification. Parameters of the optical dissector probes used to estimate neuron number were given in detail in Table I. The delineations of the areas included in the stereological

Table I. Parameters of the optical fractionator probes used to estimate neuron number

\begin{tabular}{|l|c|c|}
\hline Parameter & DG/CA3 & CA1 \\
\hline Grid spacing $(\mu \mathrm{m})$ & $300 \times 300$ & $150 \times 150$ \\
\hline Counting frame $(\mu \mathrm{m})$ & $50 \times 50$ & $50 \times 50$ \\
\hline Dissector height $(\mu \mathrm{m})$ & 12 & 12 \\
\hline
\end{tabular}


estimation of neuron number are based on the rat hippocampus atlas (48). The following regions of interest were chosen: $\mathrm{DG}, \mathrm{CA} 3$, and $\mathrm{CA} 1$.

The tissue thickness was measured at every dissector location, and the measured thickness was $15.68 \pm 2.14 \mu \mathrm{m}$ (mean $\pm \mathrm{SD}$ ). Section thickness did not differ between groups. Dissector height was $12 \mu \mathrm{m}$ and a $1-\mu \mathrm{m}$ guard zone at the top and bottom part of the section was excluded from the analysis at every step. The neurons were identified by a unique identifiable point, chosen to be the nucleolus, which had to come into focus as one focused point through the thickness of the dissector, and the nucleolus had to be inside the counting frame without touching the exclusion lines. Glial cells were not counted, and these were generally differentiated from neurons using morphological criteria, markedly the absence of a large nucleus with clear heterogeneity of chromatin. The estimated total number of neurons in each area was calculated based on the optical fractionator counts using Stereo Investigator. The Gundersen's coefficient of error (CE) values (for each individual estimate) were calculated in Stereo Investigator and a smoothness factor $m=1$ was chosen, as this is considered a suitable choice for most biological samples (57).

The mean $\mathrm{CE}$ was calculated as $\sqrt{\text { mean } \mathrm{CE}^{2}}$ and the coefficient of variation (CV) (the observed $\mathrm{CV}$ among rats) as $\mathrm{SD} /$ mean. The ratio $\mathrm{CE}^{2} / \mathrm{CV}^{2}$ was also calculated to judge the contribution of the stereological analysis to the observed group variance, as opposed to the part due to biological variance.

\section{Statistical analysis}

Two-way analysis of variance (ANOVA) followed by post hoc Bonferroni test was done to assess the differences in the rate of learning among the groups over the days during acquisition in RAM and Barnes maze task. To confirm the differences in learning rates for each group, one-way ANOVA followed by post hoc Tukey's test over the days was done during acquisition. Retention tests, for all the behavioral studies, stereological assessment of total hippocampal volume, neuron count, and all the biochemical parameters were assessed using repeated measures ANOVA followed by post hoc Tukey's multiple comparison tests. The relationship between hippocampal CA1/CA3 neuronal cells and reference/working memory errors was analyzed by Spearman's correlation coefficient. All statistical analyses were carried out using GraphPad Version 5 (Prizm; GraphPad Software Inc, San Diego, California, USA). Each value represents mean \pm standard error of mean $(n=8)$. Probability values less than 0.05 were considered statistically significant.

\section{Results}

\section{Effects of MSG on anthropometric and serum parameters}

Postnatal MSG administration resulted in increase in body weight, BMI as early as 2 months of age. Serum glucose, insulin, cholesterol, TBARs, and butyrylcholinesterase were significantly elevated in the MSG-treated rats in both age groups indicating metabolic derangement at a very early stage. Although the cholesterol and TBARs remained elevated, increase in insulin levels seen in the MSG rats was significantly reduced upon exposure to enriched housing in both the age groups (Table II).

\section{Effect of MSG on RAM performance}

Errors in four-arm baited task. MSG rats in both age groups indiscriminately entered all the arms and thus the effect of MSG significantly impaired the behavioral performance of the 


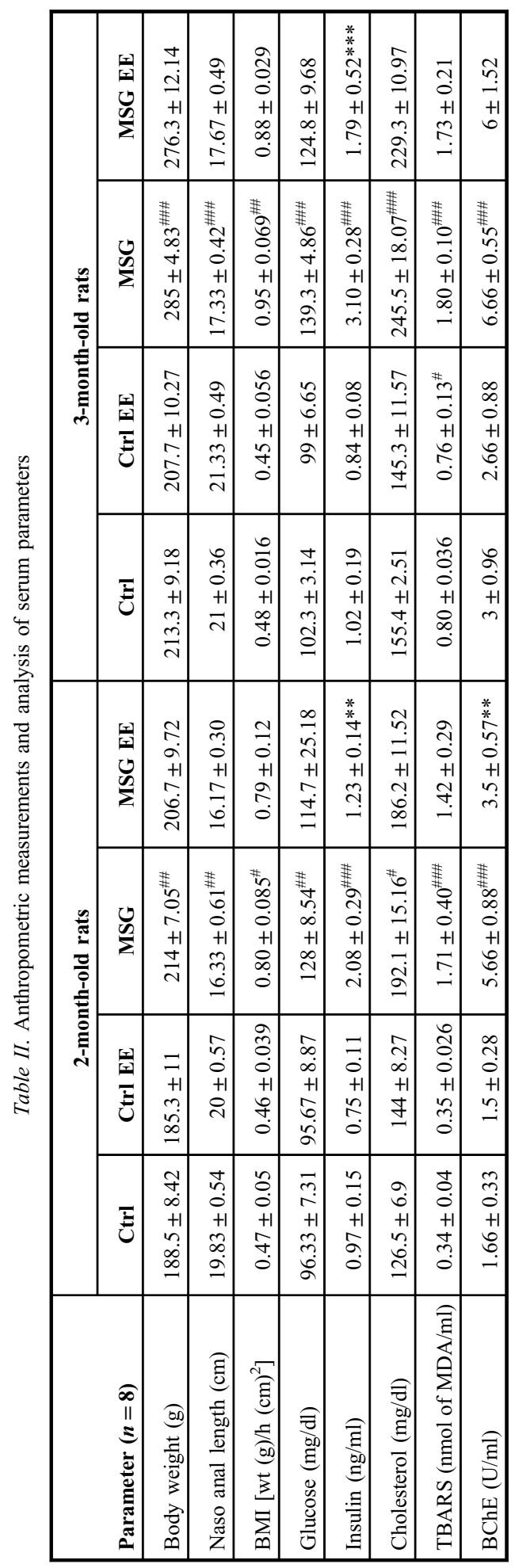

$\frac{\vec{d}}{\stackrel{\Xi}{\Xi}}$

空

离

营

茫

范

竞

近

泀

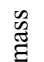

帘

离

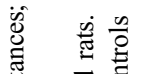

范

क

.

긍

ग

壱

율 है

ஜิ

票

氙 ठ 蓓

플 $\mathrm{D}$

का 2 के

言 $\quad * \quad 0$

政

घี

نु. 
spatial task but with training in both age groups, there were reductions in the reference memory errors (Fig. 2A and C). Entry into an unbaited arm was considered as reference memory error. MSG rats committed greater number of reference memory errors by entering into the unbaited arms. We have observed significant differences in performance across days $[2 \mathrm{M} ; F(8,252)=43.25, p<0.0001 ; 3 \mathrm{M} ; F(8,252)=39.01$, $p<0.0001]$ and between groups $[2 \mathrm{M} ; F(3,252)=91.21, p<0.0001 ; 3 \mathrm{M} ; F(3,252)=$ $119.80, p<0.0001]$. Ctrl and Ctrl EE rats made fewer reference memory errors during the acquisition trials when compared with MSG and MSG EE group rats. A significant reduction in the entries into the unbaited arms was observed from day 3 in MSG EE rats, and a significant reduction in reference memory errors was observed in the 2-month-old MSG EE group by day 9 but not in the 3-month-old MSG EE group in comparison with those in age-matched MSG rats (Fig. 2A and C). During retention, in both age groups, Ctrl and Ctrl EE rats were able to retain the memory of the learned task and performed well by committing less reference memory errors. In contrast, MSG rats committed more reference memory errors indicating impairment of implicit/non-contextual memory. MSG EE rats performed well during retention by consolidating the implicit memory learned during acquisition as reflected by less reference memory errors in comparison with age-matched MSG rats (Fig. 2E and G).

During the initial days of training in the RAM task, rats from all groups made random reentries into arms already visited. As the training continued, all the rats from the four groups showed progressive decrease in working memory errors as a measure of task learning. Reentry into an arm was considered as working memory error. There were significant differences in performance across days $[2 \mathrm{M} ; F(8,252)=52.49, p<0.0001 ; 3 \mathrm{M}$; $F(8,252)=66.35, p<0.0001]$ and between groups $[2 \mathrm{M} ; F(3,252)=79.17, p<0.0001$; $3 \mathrm{M} ; F(3,252)=66.58, p<0.0001]$ (Fig. 2B and D). Ctrl and Ctrl EE rats have shown reduction in working memory errors from days 3 and 4 in both the age groups. MSG rats at 2 months of age have shown reduction in working memory errors from day 5 during acquisition as well as during retention, which has further improved upon EE exposure. However, 3-month-old MSG rats continued to commit errors indicating aberrations in explicit memory (Fig. 2B and D). Two-month-old MSG EE rats performed well during retention by consolidating the explicit memory learned during acquisition as reflected by less working memory errors in comparison with age-matched MSG rats (Fig. 2F and $\mathrm{H}$ ). The same trend was not observed in the 3-month-old MSG EE rats as there were no significant reduction in the working memory errors indicating an age-dependent impairment in the learning and memory behavior (Fig. $2 \mathrm{~F}$ and $\mathrm{H}$ ). The latency time taken to complete the partially baited RAM task revealed significant differences among the Ctrl and MSG groups in both age groups $(p<0.0001)$. MSG rats exposed to EE have shown comparative reduction in latency in both age groups with respect to age-matched MSG rats, but significant changes were not observed (data not shown).

\section{Effect of MSG and EE on Barnes circular maze performance}

When analyzing the time taken to find the escape box in Barnes maze, during acquisition, MSG rats showed a significant increase in time (latency) in finding the escape box in comparison with the age-matched controls (Fig. 3A and C), which was effectively reduced in MSG EE groups. Similar trend of improvement in reduction in latency was appreciable in MSG EE group during retention trials (Fig. 3E and G). 

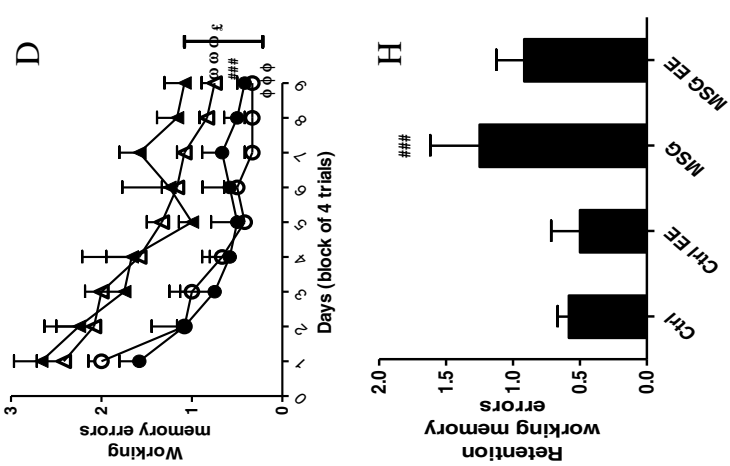

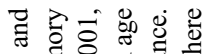

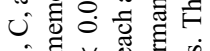

๑

¿

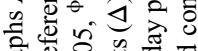

苛过空

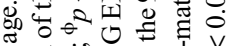

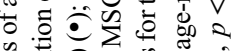

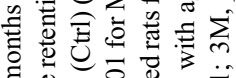

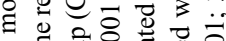
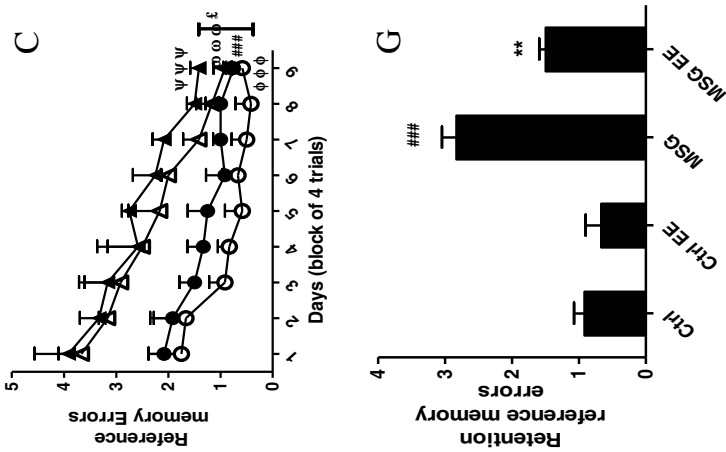

矛

뉼

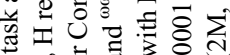

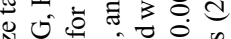

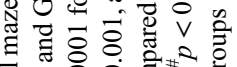

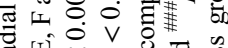

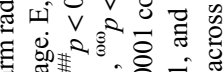

奈芯耤

过 $3^{*}$ *

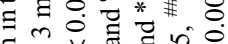

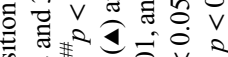

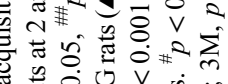

o

$\infty$

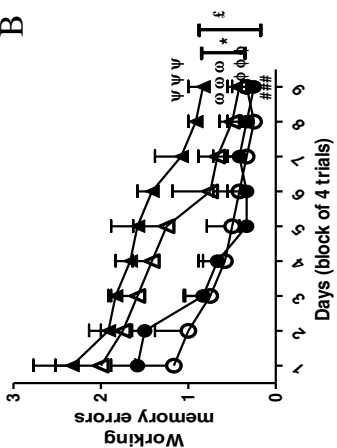

$\leftarrow$

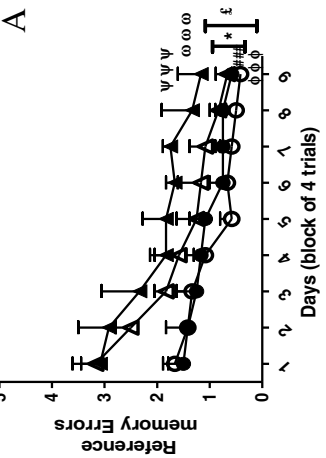

II

華

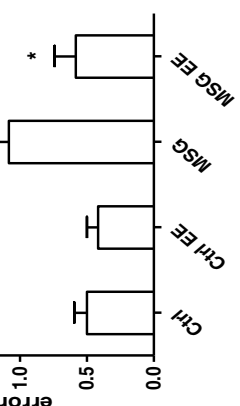

니

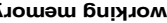
uo!̣uㅣㅏㅄ

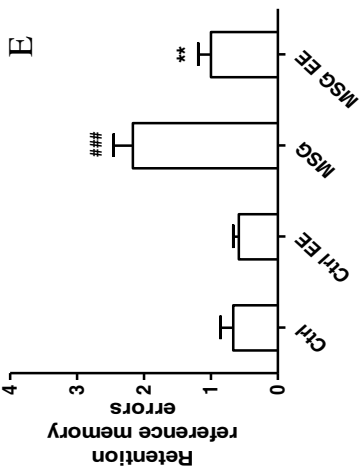

焉㻤

矛芯

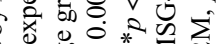

0

声记

की कू क्ड

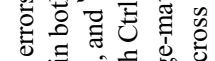

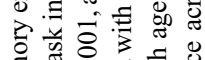

范志完

on :

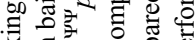

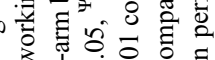

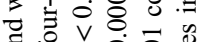

స

कै

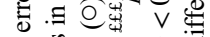

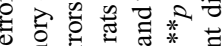

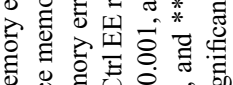

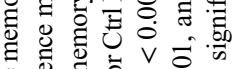

\&

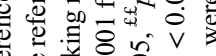

过

䒕 $\vee \vee *$

F D

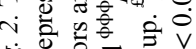

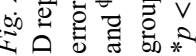



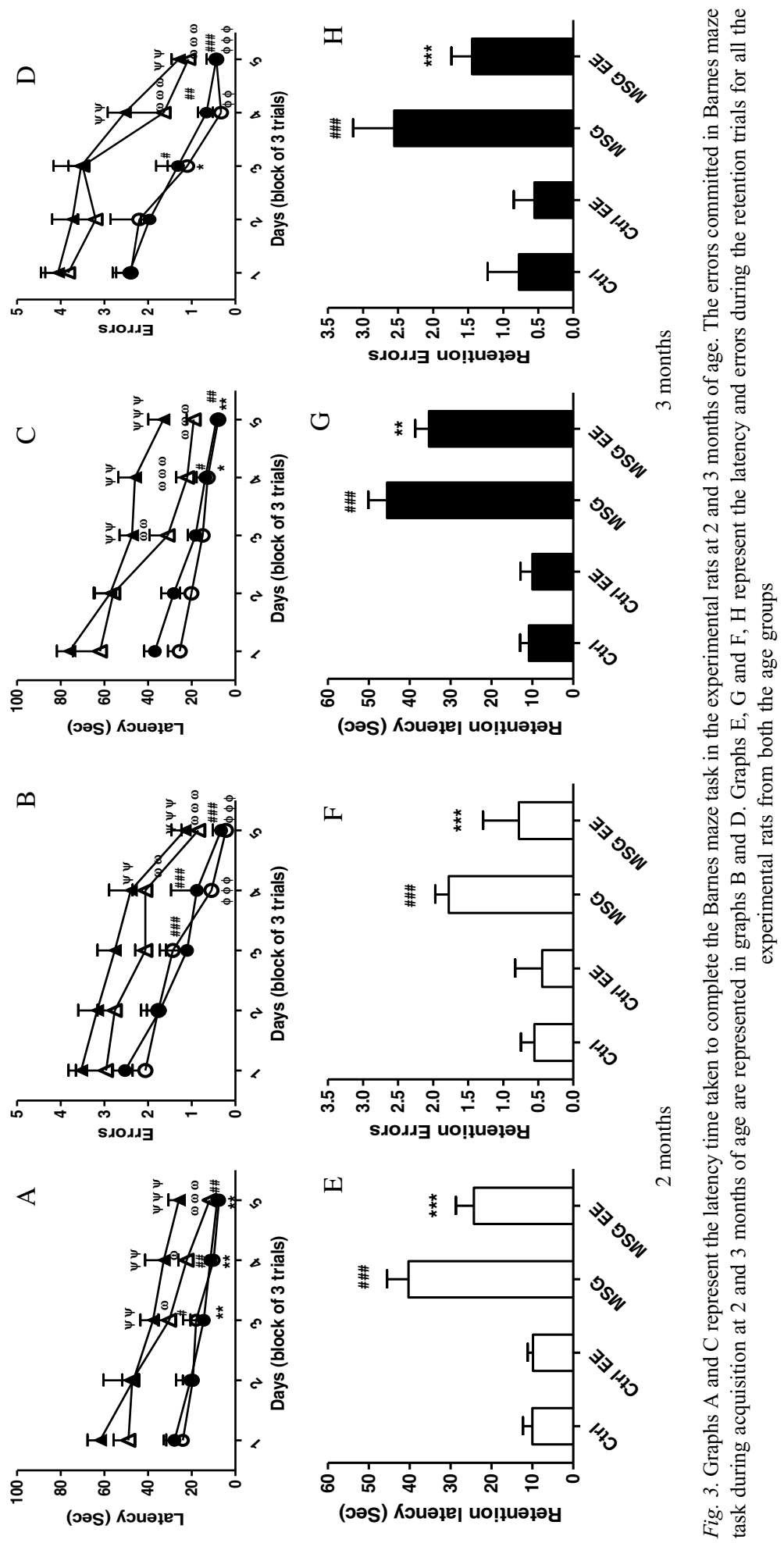
On the first day of the training session, all the rats from different groups in both age groups committed more errors during exploration of the maze (Fig. 3B and D) and by day 5, the mean number of errors to find the escape box remained significantly high in MSG rats at both age groups in comparison with Ctrl rats. Although the mean number of errors were reduced in the MSG EE groups, it was however not significant in comparison with the agematched MSG rats.

When retention trials were done after 5 days of acquisition, the memory of the escape box was consolidated in both age groups of MSG EE rats, which was evident from the reduction in the number of errors in comparison with age-matched MSG rats (Fig. 3F and $\mathrm{H}$ ).

\section{Total hippocampal volume}

The mean number of hippocampal sections per group was given in detail in Table III, and the mean total number of points per rat was 2,542 $(\min =2,019, \max =2,865)$. Shrinkage during the staining process was approximately $49 \%$. The mean coefficient of errors for the total hippocampal volume estimates ranged between 0.007 and 0.009 , using the demonstrated methods (20). Comparing the volume in the dorsal hippocampus between left and right hemispheres indicated no significant difference. Therefore, the data were interpreted as total hippocampal volume of the dorsal hippocampus. Analysis of total hippocampal volume by ANOVA indicated that there was a significant reduction in volume in the MSG rats in both age groups in comparison with the age-matched controls. Significant increase in volume with $11.3 \%$ was noticed in the 2-month-old MSG EE group in comparison with the MSG group. In 3-month-old rats, $10.09 \%$ increase in volume was observed in MSG EE group in comparison with the MSG group. However, EE treatment to Ctrl rats did not elicit significant increase in the hippocampal volume (Fig. 4).

\section{Estimation of neuron number}

The stereological estimates of neuronal number showed $18.21 \%$ reduction in the 2 -month-old MSG group and $20.19 \%$ reduction in the 3-month-old MSG rats in comparison with the controls (Fig. 5A, a) in the CA1 region. In the DG region, $15.4 \%$ reduction of neurons in the case of 2-month-old MSG rats and $18.75 \%$ in the 3-month-old MSG rats were noticed in comparison with the age-matched control rats. In the CA3 region, there was a $13.61 \%$ reduction in the neuron number in the 2-month-old MSG group and $19.17 \%$ reduction in the 3-month-old MSG rats in comparison with the controls (Fig. 5B, b). EE-treated 3-month-old MSG rats showed significant increase in neuron number $(10.3 \%)$ in the DG region of hippocampus in comparison with the age-matched MSG rats. A significant increase in neuron number was seen in 2-month-old MSG EE rats (11.04\%) in the CA3 region of hippocampus in comparison with the age-matched MSG rats (Fig. 5B). In CA1 region, a significant increase in neuron number was observed in 2-month- (15.61\%) (Fig. 5A) and 3-month-old MSG EE groups (12.64\%) (Fig. 5a) in comparison with that in age-matched MSG rats (Fig. 5A, a). The CE of the stereological estimates was low in all groups $(<0.1)$, whereas the observed $\mathrm{CV}$ was more variable, ranging from 0.12 to 0.23 (Table III). The $\mathrm{CE}^{2} / \mathrm{CV}^{2}$ ratio was calculated for each group, and indicates that the overall biological contribution to the variance is larger than the contribution from the stereological procedure (Table III).

Correlation between hippocampal neuron cells and reference/working memory errors Evaluation of the Ctrl, MSG, and MSG EE rats revealed a negative correlation (Fig. 6) between the number of hippocampal CA3/CA1 neuronal cells and reference/working 


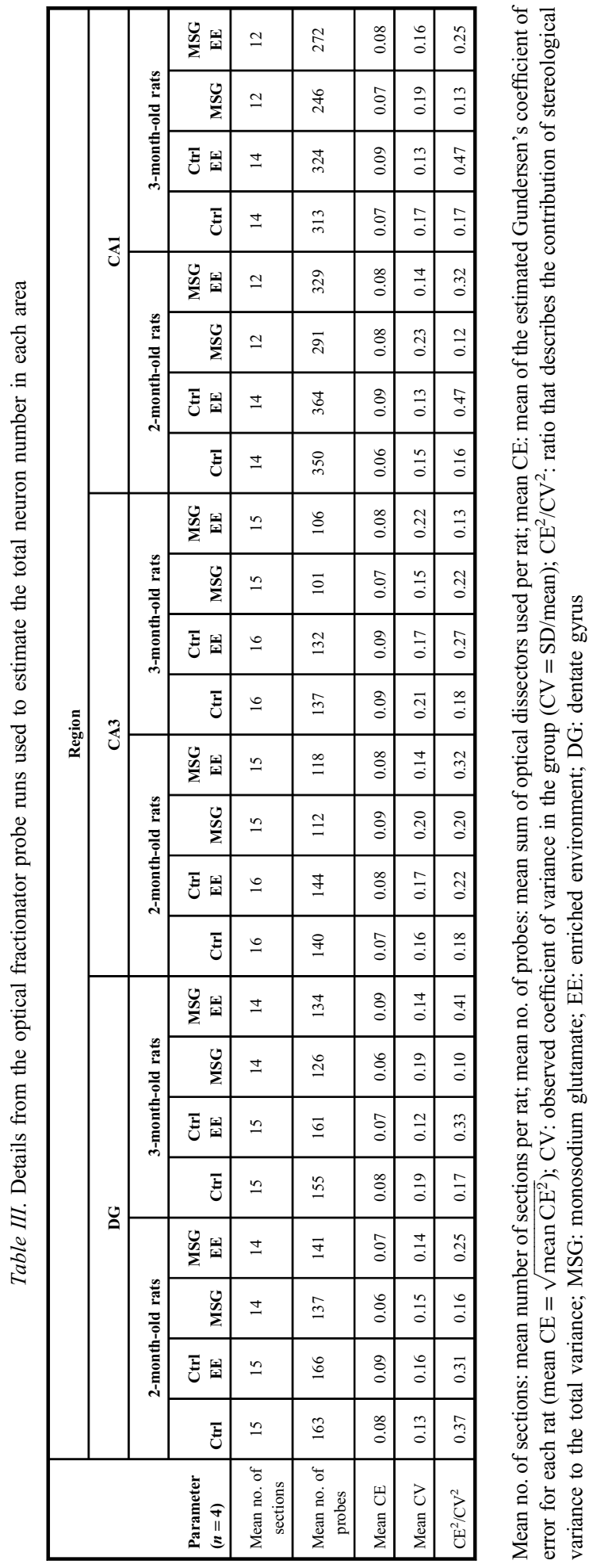




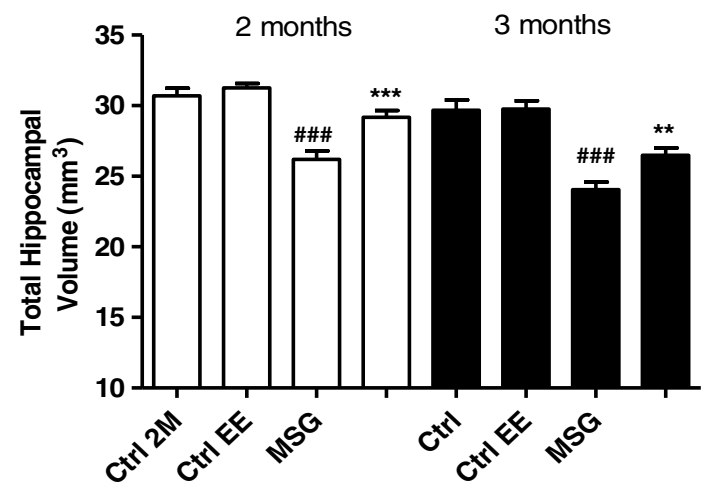

Fig. 4. Changes in total hippocampal volume. Data presented as mean $\pm \mathrm{SD}$. $* p<0.05$,

$* * p<0.001$, and $* * * p<0.0001$ compared with age-matched MSG-treated rats. ${ }^{\#} p<0.05$,

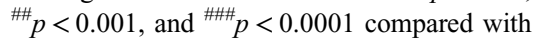
age-matched controls

memory errors in the RAM task $(r=-0.86 ; p=0.026$; Ctrl vs. MSG) $(r=-0.92 ; p=0.002$; MSG vs. MSG EE).

\section{Discussion}

In this study, we demonstrated the cognitive deficits in the postnatal MSG-induced obese rats as early as 2 months of age with hippocampal neuronal loss and insulin resistance in the periphery. As a non-invasive therapeutic approach, we studied the effects of an EE protocol on obesity-associated brain changes. EE is a housing condition, which provided a combination of social (living together with other rats) and physical stimulation (novel objects, tunnels, and slides provided in the cage) to the MSG rats. Short-term EE paradigm contributed to increased insulin sensitivity with a significant functional recovery in the MSG EE rats in both 2- and 3-month age groups. The behavioral recovery in the partially baited RAM task was more prominent in the 2-month-old MSG EE group. Although there was improvement in the performance in the 3-month-old MSG EE group, it was however not comparable with the Ctrl rats. These results implicate the importance of critical therapeutic window period in reducing the damage caused by the postnatal insults by EE in improving the neuronal plasticity.

Using behavioral and histological studies, we investigated cognitive impairment associated with hippocampal neuronal loss in the MSG-treated rats. Hippocampus is involved in establishing a cognitive map of environment (44). The hippocampus has been shown to suffer cell loss at early stages in patients with AD (19), and neuronal loss in CA1/ hippocampus proper has been shown in both human patients (57), as well as in mice models (9) of AD. Comparing number of neurons in DG, CA3, and CA1 of the dorsal hippocampus between left and right hemispheres indicated no significant difference. Therefore, the data were interpreted as total number of neurons in each region of the dorsal hippocampus. The delineation of the areas of interest for estimation of neuron number was based on the rat hippocampus atlas (48). Cresyl violet stained slides were proved to be very feasible in estimating the neuron number using unbiased stereological method by Zhu et al. (58), and hence, this method was considered in this study.

Since the spatial working memory is affected in severely demented and AD cases (26), in this study, RAM and Barnes maze tasks were employed to assess the spatial learning and memory functions in the experimental rats. Reference memory is a type of memory used to store and retain memory of events that are constant throughout the testing procedure (the 

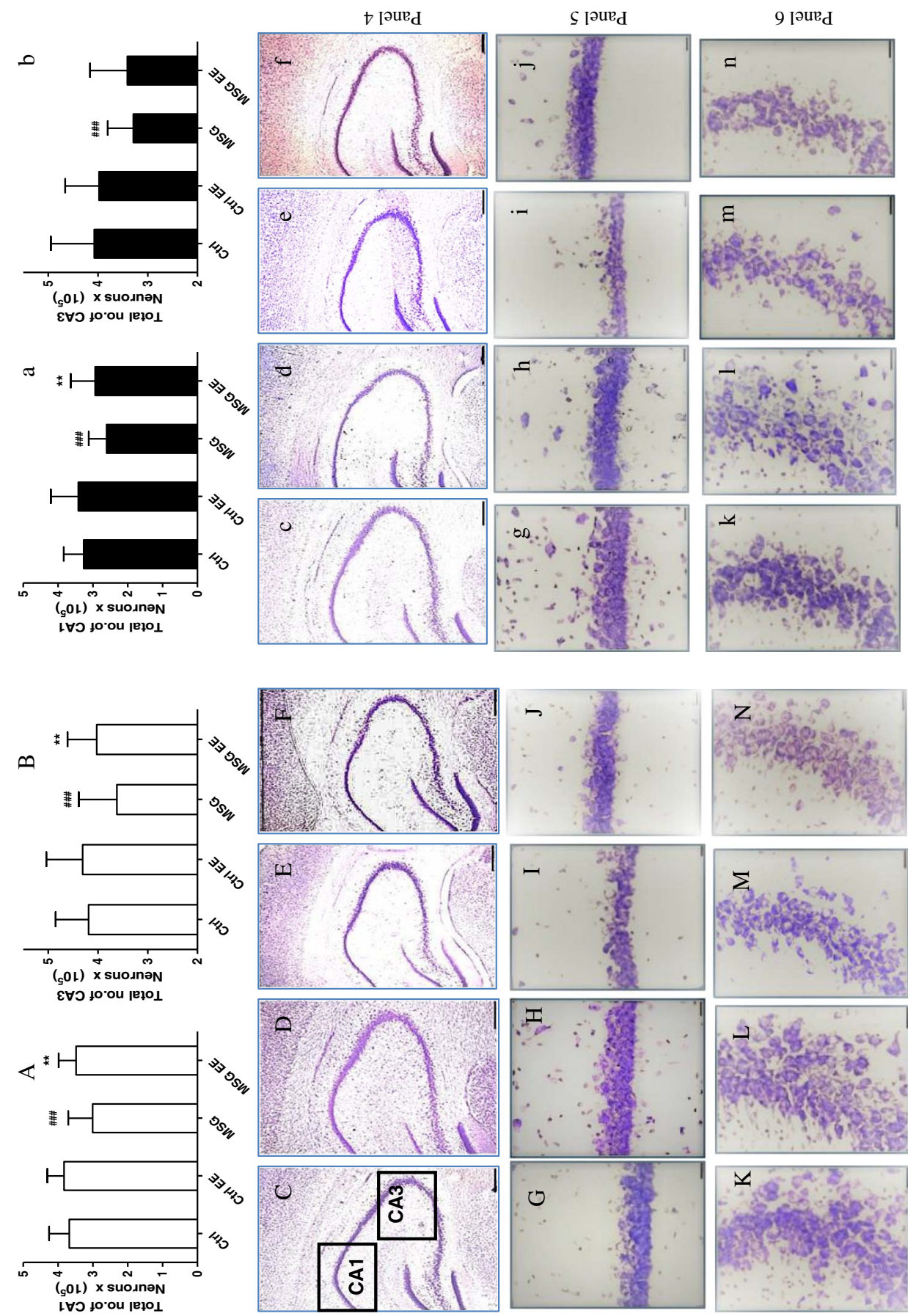

I [our $\mathrm{d}$

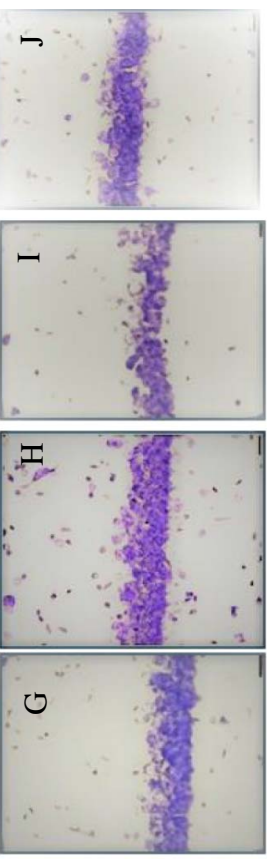

乙 Iourd
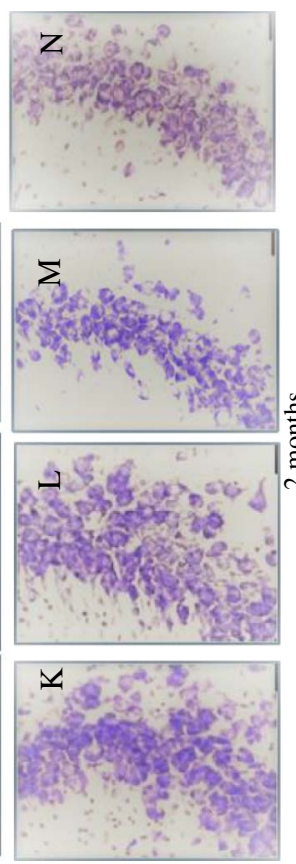

$\mathcal{E}$ [əux

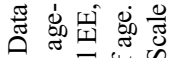
DE $E$ 员 (5)

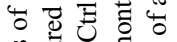
을 을

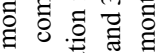
क

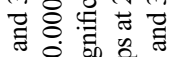
ป $)$ तथ 2 ह 茟 草学

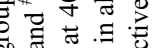

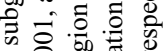

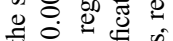
总 鞋芯芯

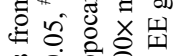
운 응워

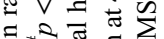

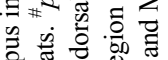
敬论 త్ 己

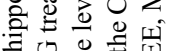

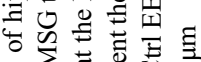

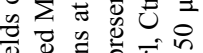

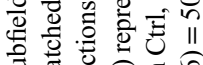
क 范节. (2) थิ 过 घ తิ 文。 $\varangle 8$ 용 芯 * $\Xi *$ 苛

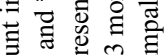
ठิ 웡 $\exists \vee$ 巳 추웡 눙 चี

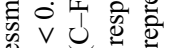
की की

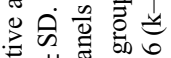
试 +1 데 둥

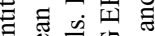

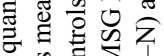

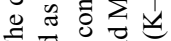

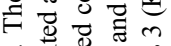
i

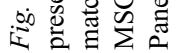



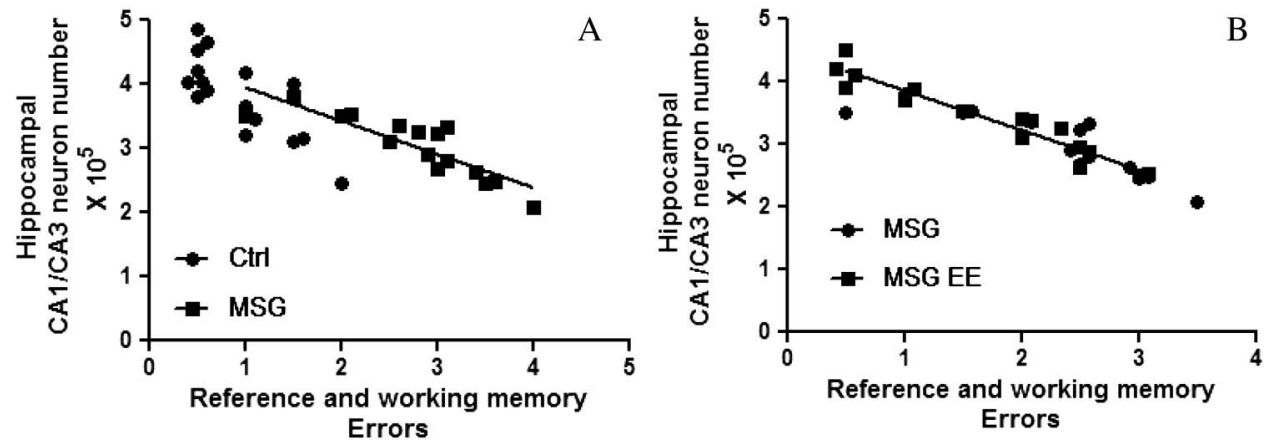

Fig. 6. Correlation between reference/working memory errors and hippocampal CA1/CA3 neuron number. A negative correlation $(r=-0.86 ; p=.026$; Ctrl vs. MSG in panel A) $(r=-0.92 ; p=.002$; MSG vs. MSG EE in Panel B) was observed. Results represent both sides of hippocampus

sequence of baited arms) (46) and involves the formation of spatial or cognitive maps of the maze and its surrounding environment (15). Reference memory errors indicate implicit memory (non-contextual memory). Explicit memory, on the other hand, can be measured in the RAM task by assessing the number of times a rat goes back to an arm where it has already received food. Working memory refers to a set of processes needed to remember cues that differ from trial to trial in the maze, which implicates the explicit memory. In this study, both reference and working memory components were disrupted in the MSG rats in the RAM task (Fig. 2). In addition, MSG-induced obesity disrupted the place orientation abilities in Barnes maze task in rats with increase in latency and errors in both the age groups (Fig. 3). The loss of neurons in the hippocampal region (CA1 and CA3) (Fig. 5) in the MSG rats is the best current pathologic correlate of cognitive decline (Figs 2 and 3) and with the EE exposure in both age groups, there was a significant increase in CA1 neuron number contributing to improved learning during the acquisition trials and memory consolidation during the retention trials with respect to the spatial reference memory task (Fig. 2A and C). Indeed, the dorsal hippocampus seems to be highly involved in spatial learning (41). Moreover, all hippocampal subregions are highly interconnected, suggesting that, individually, they may serve discrete computational functions. The functionality of the hippocampal subregions can be dissociated behaviorally using different mazes for assessing the spatial working memory. New neurons are born throughout life in the DG region of hippocampus and several hypotheses have been developed to explain how new neurons might contribute to hippocampal function (30). It has been proposed by Aimone et al. (1) that the new neurons, which are young when events occur, have a greater role in encoding and storage and in temporally relating one event to another. In this study, there was a marginal increase in neuron number in the DG region of EE exposed 2-month-old MSG rats and this can be correlated to the behavioral recovery in the RAM and Barnes maze tasks. The same phenomenon was not attributed to the 3-month-old MSG rats exposed to EE. Although we observed a significant increase in neuron number in the DG region, improvement in the spatial working memory was not observed highlighting the functional importance of the other hippocampal subregions (CA3 and CA1) in the behavioral studies. The CA3 network has been allocated the capacity to enable rapid acquisition of unique associations and to store patterned information received from the DG or directly from the entorhinal cortex for a short period of time (50). Accordingly, we can hypothesize a crucial role of the CA3 network in contextual memory 
acquisition. The present results are consistent with previous studies conducted by Handelmann and Olton (21) and Jarrard (28) where they showed that rats with CA3 lesions are impaired in spatial working memory, even though there was significant neurogenesis observed in the DG region of hippocampus. EE exposure to MSG rats at 2 months has shown improvement in the spatial working memory task during the retention trials in comparison with the age-matched MSG rats (Fig. 2F), whereas the same EE paradigm in the 3-month-old MSG EE rats has not shown any significant improvement (Fig. 2H), and this behavioral data can be correlated with the stereology results where there was a significant increase in neuron number in the CA3 region of 2-month-old MSG rats exposed to EE but not in the 3-month-old MSG EE group. CA1 is mainly involved in the information-processing function and it has no autoassociative properties and it is considered as the major output structure of the hippocampus. Indeed, the CA1 area might be instrumental in recognizing the novelty or familiarity of an object or context (42). Therefore, this area might be rather involved in the consolidation and retrieval of recent contextual memory than in its processing. CA1 pyramidal cell axons primarily target the subiculum (3) and thus represent the final relay in a synaptic loop between the entorhinal cortex and hippocampus. With the EE exposure, MSG rats in both age groups have shown a significant increase in CA1 neuron number, which contributed to improved learning and memory in the spatial reference memory task (Fig. 2A and $\mathrm{C}$ ).

We have observed weak behavioral recovery following repeated trials in the RAM in the MSG rats (Fig. 2). Intensive training with repeated trials helps to overcome the behavioral deficits and it can be due to the involvement of other structures like pre/para subiculum, which were shown to be essential for spatial memory (49). The behavioral recovery observed in MSG EE rats in this study also probably suggests the possibility of an alternative strategy that the rats used to make the right choices. Our results showed an effect of the environmental stimulation on cognitive flexibility and reduced neuronal loss in the hippocampus of the MSG rats exposed to EE. The novelty and efficacy of the short-term-enriched housing conditions on the behavioral performance accompanying the phenomena of improving the insulin sensitivity in the MSG-induced rats has also been elucidated. The impact of the obesity followed by insulin-resistant state on the CNS, specifically on limbic structures, has been extensively studied by several groups and they concluded that these peripheral disturbances can lead to increased risk of cognitive dysfunction and dementia (54). Disruptions to insulin levels or insulin signaling in the brain can contribute to neuronal dysfunction, degeneration, and cognitive impairment (5). The beneficial effects of EE on the hippocampus have been described in rat models of physiological and pathological conditions (43), counting aging (31) and $\mathrm{AD}$ (12) among other situations. It was proved by Rovio et al. (51) in human subjects of dementia and $\mathrm{AD}$ with an improvement in cognition following an active lifestyle, which included a combination of social, physical, and cognitive components. The increase in neuron number in the CA1 and CA3 regions of the MSG EE 2M group and corresponding correlations between working memory errors established a link between increase in neuron number in the CA1 and CA3 subregions and working memory. This was supported in this study with the correlation analysis where the negative correlation between the number of CA1 and CA3 hippocampal neurons evaluated by Spearman's correlation coefficient indicated that neonatal MSG administration caused loss of hippocampal neuronal cells, and this loss has contributed to increase in reference and working memory errors in the RAM task (Fig. 6A). With the EE exposure, MSG EE rats have shown recovery with reduced reference and working memory errors and with an increase in the number of hippocampal CA1 and 
CA3 neuronal cells, which was confirmed by the negative correlation that was evaluated between the MSG and MSG EE rats (Fig. 6B).

It is pertinent to mention at this juncture that although the RAM and Barnes maze are designed to assess the spatial learning abilities, one cannot generalize that these mazes utilize the same environmental visuospatial cues to form cognitive maps, rather the mazes differ in their search strategies and tap a variety of sources of non-spatial information in addition to the visuospatial cues for the construction of spatial maps (55). Barnes maze utilizes an aversive learning strategy when compared with the reward task in RAM. The nature of errors committed is also different in both tasks. The RAM explicitly requires working memory, and the hippocampus is essential for mediating working memory. Barnes maze helps to study the spatial localization component of hippocampal functioning. The behavioral performances of rats in different mazes cannot be correlated as they use different strategies to complete the given maze task (55). EE might not reverse the damaged function, but it may influence the unaffected abilities of the rat (16). We found such behavioral enhancement even in the Barnes maze performance in the MSG rats following enrichment during retention trials. The errors committed by the MSG EE rats following enrichment were comparable with the Ctrl rats exposed to standard housing at 2 months of age, whereas at 3 months of age, MSG EE rats' performance in the four-arm baited task was not comparable with the control rats and in this case, may be some changes in the EE paradigm with increase in number of days might have helped. The intensive training in the RAM with the EE exposure stimulated the hippocampal circuits in $2 \mathrm{M}$ MSG EE rats to a greater extent with complete functional recovery. At 3 months, the same EE paradigm has shown a lesser degree of recovery in comparison with the 2-month-old MSG EE group, highlighting the importance of critical window period where the experience-dependent plasticity reduces with increase in age. The enhanced insulin sensitivity observed in EE-exposed rats in this study might have mediated the effects of EE on hippocampal synaptology. This requires further studies. Additional studies using specific neuronal markers for neurogenesis need to be investigated in detail.

In summary, our findings elucidate the importance of EE and the critical therapeutic window period on behavioral recovery when considered at an early stage (45 days) in the postnatal MSG-treated rats than at a later stage. These observations are in agreement with the reported literature that EE intervention must occur early in disease course, prior to substantial cognitive deficits (18). These findings hold potential for translation to clinical research to study the influence of EE on individuals in the prodromal or preclinical stage of AD or in genetically susceptible individuals. However, this warrants further large-scale studies.

\section{Acknowledgements}

Financial assistance to SS from Council of Scientific and Industrial Research, New Delhi [Grant no. 37 (1607) 13/ EMR-II] is gratefully acknowledged. SM is fellow of WOS-A scheme funded by Department of Science and Technology, New Delhi, India.

\section{REFERENCES}

1. Aimone JB, Wiles J, Gage FH: Potential role for adult neurogenesis in the encoding of time in new memories. Nat. Neurosci. 9, 723-727 (2006)

2. Ali AE, Wilson YM, Murphy M: A single exposure to an enriched environment stimulates the activation of discrete neuronal populations in the brain of the fos-tau lacZ and voluntary exercise massively increase neurogenesis in the adult hippocampus via dissociable pathways. Hippocampus 16, 250-260 (2009) 
3. Amaral DG, Dolorfo C, Alvarez-Royo P: Organization of CAl projections to the subiculum: a PHA-L analysis in the rat. Hippocampus 1, 415-436 (1991)

4. Andre C, Dinel AL, Ferreira G, Laye S, Castanon N: Diet-induced obesity progressively alters cognition, anxiety-like behavior and lipopolysaccharide-induced depressive-like behavior: focus on brain indoleamine 2, 3dioxygenase activation. Brain Behav. Immunol. 41, 10-21 (2014)

5. Apostolatos A, Song S, Acosta S, Peart M, Watson JE, Bickford P, Cooper DR, Patel NA: Insulin promotes neuronal survival via the alternatively spliced protein kinase C $\delta$ II isoform. J. Biol. Chem. 287, 9299-9310 (2012)

6. Bindu B, Rekha J, Kutty BM: Post insult enriched housing improves the 8-arm radial maze performance but not the Morris water maze task in ventral subicular lesioned rats. Brain Res. 1063, 121-131 (2005)

7. Boitard C, Cavaroc A, Sauvant J, Aubert A, Castanon N, Laye S, Ferriera G: Impairment of hippocampaldependent memory induced by juvenile high-fat diet in take is associated with enhanced hippocampal inflammation in rats. Brain Behav. Immunol. 40, 9-17 (2014)

8. Bonda DJ, Stone JG, Torres SL, Siedlak SL, Perry G, Kryscio R, Jicha G, Casadesus G, Smith MA, Zhu X, Lee $\mathrm{H}$ : Dysregulation of leptin signalling in Alzheimer disease: evidence for neuronal leptin resistance. J. Neurochem. 128, 162-172 (2014)

9. Bouter Y, Dietrich K, Wittnam JL, Rezaei-Ghaleh N, Pillot T, Papot-Couturier S, Lefebvre T, Sprenger F, Wirths O, Zweckstetter M, Bayer TAP: N-truncated amyloid $\beta$ (A $\beta$ ) 4-42 forms stable aggregates and induces acute and long-lasting behavioral deficits. Acta Neuropathol. 126, 189-205 (2013)

10. Brotons-Mas JR, O’Mara S, Sanchez-Vives MV: Neural processing of spatial information: what we know about place cells and what they can tell us about presence. Presence 15, 485-499 (2006)

11. Buschler A, Goh JJ, Manahan-Vaughan D: Frequency-dependency of NMDA receptor-dependent synaptic plasticity in the hippocampal CA1 region of freely behaving mice. Hippocampus 22, 2238-2248 (2012)

12. Costa DA, Cracchiolo JR, Bachstetter AD, Hughes TF, Bales KR, Paul SM, Mervis RS, Arendash GW, Potter H: Enrichment improves cognition in $\mathrm{AD}$ mice by amyloid-related and unrelated mechanisms. Neurobiol. Aging 28, 831-844 (2007)

13. Dahl AK, Hassing LB, Fransson EI, Gatz M, Reynolds CA, Pedersen NL: Body mass index across mid-life and cognitive change in late life. Int. J. Obesity 37, 296-302 (2013)

14. Dawson R, Pelleymounter MA, Millard WJ, Liu S, Eppler B: Attenuation of leptin-mediated effects by monosodium glutamate-induced arcuate nucleus damage. Am. J. Physiol. Endocrinol. Metab. 273, E202-E206 (1997)

15. Eichenbaum H, Stewart C, Morris RG: Hippocampal representation in place learning. J. Neurosci. 10, 3531-3542 (1990)

16. Einon DF, Morgan MJ, Will BE: Effects of post-operative environment on recovery from dorsal hippocampal lesions in young rats: tests of spatial memory and motor transfer. J. Exp. Psychol. 32, 137-148 (1980)

17. Ellman GL, Courtney KD, Andres V, Featherstone RM: A new and rapid colorimetric determination of acetylcholinesterase activity. Biochemical Pharmacol. 7, 88-95 (1961)

18. Gehres SW, Rocha A, Leuzy A, Loss CM, Viola GG, Zimmer ER: Cognitive intervention as an early pharmacological strategy in Alzheimer's disease: a translational perspective. Front. Aging Neurosci. 8, 260 (2016)

19. Gomez-Isla T, Price JL, McKeel DW Jr, Morris JC, Growdon JH, Hyman BT: Profound loss of layer II entorhinal cortex neurons occurs in very mild Alzheimer's disease. J. Neurosci. 16, 4491-4500 (1996)

20. Gundersen HJ: Stereology of arbitrary particles. A review of unbiased number and size estimators and the presentation of some new ones, in memory of William R. Thompson. J. Microscopy 143, 3-45 (1986)

21. Handelmann GE, Olton DS: Spatial memory following damage to hippocampal CA3 pyramidal cells with kainic acid: impairment and recovery with preoperative training. Brain Res. 217, 41-58 (1981)

22. Hensch TK, Bilimoria PM: Re-opening windows: manipulating critical periods for brain development. Cerebrum 11, 1-18 (2012)

23. Hlinak Z, Gandalovicova D, Krejci I: Behavioral deficits in adult rats treated neonatally with glutamate. Neurotoxicol. Teratol. 27, 465-473 (2005)

24. Horvath G, Reglodi D, Vadasz Gy, Farkas J, Kiss P: Exposure to enriched environment decreases neurobehavioural deficits induced by neonatal glutamate toxicity. Int. J. Mol. Sci. 14, 19054-19066 (2013)

25. Hu YS, Xu P, Pigino G, Brady ST, Larson J, Lazarov O: Complex environment experience rescues impaired neurogenesis, enhances synaptic plasticity, and attenuates neuropathology in familial Alzheimer's disease-linked APPswe/PS1DeltaE9 mice. FASEB J. 24, 1667-1681 (2010)

26. Huntley J, Bor D, Hampshire A, Owen A, Howard R: Working memory task performance and chunking in early Alzheimer's disease. Br. J. Psychiatry 198, 398-403 (2011) 
27. Hwang LL, Wang CH, Li TL, Chang SD, Lin LC, Chen CP, Chen CT, Liang KC, Ho IK, Yang WS, Chiou LC: Sex differences in high-fat diet-induced obesity, metabolic alterations and learning, and synaptic plasticity deficits in mice. Obesity 18, 463-469 (2010)

28. Jarrard LE: Selective hippocampal lesions and behavior: effects of kainic acid lesions on performance of place and cue tasks. Behav. Neurosci. 97, 873-889 (1983)

29. Kanoski SE, Davidson TL: Western diet consumption and cognitive impairment: Links to hippocampal dysfunction and obesity. Physiol. Behav. 103, 59-68 (2011)

30. Kempermann G: The neurogenic reserve hypothesis: what is adult hippocampal neurogenesis good for? Trends Neurosci. 31, 163-169 (2008)

31. Kempermann G, Gast D, Gage FH: Neuroplasticity in old age: sustained fivefold induction of hippocampal neurogenesis by long term environmental enrichment. Ann. Neurol. 52, 135-143 (2002)

32. Kiss P, Hauser D, Tamas A, Lubics A, Racz B, Horvath ZS, Farkas J, Zimmermann F, Stepien A, Lengvari I, Reglodi D: Changes in open-field activity and novelty-seeking behaviour in periadolescent rats neonatally treated with monosodium glutamate. Neurotoxicity Res. 12, 85-93 (2007)

33. Leger M, Bouet V, Freret T, Darmaillacq AS, Dacher M, Dauphin F, Boulouard M, Schumann-Bard P: Environmental enrichment improves recent but not remote memory in association with a modified brain metabolic activation profile in adult mice. Behav. Brain Res. 228, 22-29 (2012)

34. Madhavadas S, Kapgal VK, Kutty BM, Subramanian S: The neuroprotective effect of dark chocolate in monosodium glutamate induced non-transgenic Alzheimer disease model rats: biochemical, behavioral and histological Studies. J. Dietary Suppl. 13, 449-460 (2016)

35. Madhavadas S, Kutty BM, Subramanian S: Amyloid beta lowering and cognition enhancing effects of ghrelin receptor analog [D-Lys (3)] GHRP-6 in rat model of obesity. Indian J. Biochem. Biophys. 51, 257-262 (2014)

36. Madhavadas S, Subramanian S: Combination of Spirulina with glycyrrhizin prevents cognitive dysfunction in aged obese rats. Indian J. Pharmacol. 47, 39-44 (2015)

37. Matyskova R, Maletinska L, Maixnerova J, Pirnik Z, Kiss A, Zelenza B: Comparison of the obesity phenotypesrelated to monosodium glutamate effect on arcuate nuleus and/or the high fat diet feeding in C57BL/6 and NMRI mice. Physiol. Res. 57, 727-734 (2008)

38. McQuaid RJ, Audet MC, Jacobson-Pick S, Anisman H: Environmental enrichment influences brain cytokine variations elicited by social defeat in mice. Psychoneuroendocrinology 38, 987-996 (2013)

39. Mitra R, Sapolsky RM: Short-term enrichment makes male rats more attractive, more defensive and alters hypothalamic neurons. PLoS One 7, e36092 (2012)

40. Morris MJ, Tortelli CF, Filippis A, Proietto J: Reduced BAT function as a mechanism for obesity in the hypohagic, neuropeptide Y-deficient monosodium glutamate-treated rat. Regul. Pept. 75, 441-447 (1998)

41. Moser MB, Moser EI, Forrest E, Andersen P, Morris RG: Spatial learning with a minislab in the dorsal hippocampus. Proc. Natl. Acad. Sci. U S A 92, 9697-9701 (1995)

42. Nakazawa K, McHugh TJ, Wilson MA, Tonegawa S: NMDA receptors, place cells and hippocampal spatial memory. Nat. Rev. Neurosci. 5, 361-372 (2004)

43. Nithianantharajah J, Hannan AJ: Enriched environments, experience dependent plasticity and disorders of the nervous system. Nat. Rev. Neurosci. 7, 697-709 (2006)

44. O’Keefe J, Nadel L (1978): The Hippocampus as a Cognitive Map, Oxford University Press, London

45. Olney JW: Brain lesions, obesity, and other disturbances in mice treated with monosodium glutamate. Science 164, 719-721 (1969)

46. Olton DS, Papas BC: Spatial memory and hippocampal function. Neuropsychologia 17, 669-682 (1979)

47. Pang TY, Renoir T, Du X, Lawrence AJ, Hannan AJ: Depression-related behaviours displayed by female C57BL/6J mice during abstinence from chronic ethanol consumption are rescued by wheel-running. Eur. J. Neurosci. 37, 1803-1810 (2013)

48. Paxinos G, Watson C (2004): The Rat Nervous Coordinates, The New Coronal Set, Elsevier, New York.

49. Riegert C, Galani R, Heilig S, Lazarus C, Cosquer B, Cassel JC: Electrolytic lesions of the ventral subiculum weakly alter spatial memory but potentiate amphetamine-induced locomotion. Behav. Brain Res. 152, 23-34 (2004)

50. Rolls ET (1996): Roles of long term potentiation and long term depression in neuronal network operations in the brain. In: Cortical Plasticity, eds Fazeli MS, Collingridge GL, Oxford Press, Oxford, pp. 223-250

51. Rovio S, Kareholt I, Helkala EL, Viitanen M, Winblad B, Tuomilehto J, Soininen H, Nissinen A, Kivipelto M: Leisure-time physical activity at midlife and the risk of dementia and Alzheimer's disease. Lancet Neurol. 4, 705-711 (2005) 
52. Saari MJI, Fong S, Shivji A, Armstrong JN: Enriched housing masks deficits in place navigation induced ny neonatal monosodium glutamate. Neurotoxicol. Teratol. 12, 29-32 (1990)

53. Sahay A, Scobie KN, Hill AS, O’Carroll CM, Kheirbek MA, Burghardt NS, Fenton AA, Dranovsky A, Hen R: Increasing adult hippocampal neurogenesis is sufficient to improve pattern separation. Nature 472, 466-470 (2011)

54. Schuff N, Woerner N, Boreta L, Kornfield T, Shaw LM, Trojanowski JQ, Thompson PM, Jack CR Jr, Weiner MW: MRI of hippocampal volume loss in early Alzheimer's disease in relation to ApoE genotype and biomarkers. Brain 132, 1067-1077 (2009)

55. Sutherland RJ, Whishaw IQ, Kolb B: Contributions of cingulated cortex to two forms of spatial learning and memory. J. Neurosci. 8, 1863-1872 (1988)

56. Tanti A, Westphal WP, Girault V, Brizard B, Devers S, Le Guisquet AM, Surget A, Belzung C: Regiondependent and stage-specific effects of stress, environmental enrichment and antidepressant treatment on hippocampal neurogenesis. Hippocampus 23, 797-811 (2013)

57. West MJ, Coleman PD, Flood DG, Troncoso JC: Differences in the pattern of hippocampal neuronal loss in normal ageing and Alzheimer's disease. Lancet 344, 769-772 (1994)

58. Zhu Y, Liu F, Zou X, Torbey M: Comparison of unbiased estimation of neuronal number in the rat hippocampus with different staining methods. J. Neurosci. Meth. 254, 73-79 (2015) 